\title{
Optimal Dosing of Breast Cancer Chemotherapy using Robust MPC Based on Linear Matrix Inequalities
}

\author{
Pornchai Bumroongsri $^{1, \mathrm{a}, *}$ and Soorathep Kheawhom ${ }^{2, \mathrm{~b}}$. \\ 1 Department of Chemical Engineering, Faculty of Engineering, Mahidol University, Phuttamonthon 4 \\ Road, Salaya, Nakhon Pathom, 73170, Thailand. \\ 2 Computational Process Engineering, Department of Chemical Engineering, Faculty of Engineering, \\ Chulalongkorn University, Phayathai Road, Patumwan, Bangkok, 10330, Thailand. \\ E-mail: apornchai.bum@mahidol.ac.th (Corresponding author), bsoorathep.k@chula.ac.th
}

\begin{abstract}
In this paper, we consider an application of robust model predictive control to optimal dosing of breast cancer chemotherapy. The model-patient mismatch is handled by computing an ellipsoidal invariant set containing the measured patient's states at each sampling time. An optimal dose of chemotherapeutic agent is obtained by solving a convex optimization problem subject to linear matrix inequalities. In the case study of simulated patients, the results show that the tumor volume can be reduced to a specified target with up to $30 \%$ model-patient mismatch. Moreover, the robust model predictive control algorithm can achieve better treatment results as compared with the nonlinear model predictive control algorithm while the on-line computational time is significantly reduced.
\end{abstract}

Keywords: Robust model predictive control, breast cancer chemotherapy, model-patient mismatch, linear matrix inequalities.

ENGINEERING JOURNAL Volume 19 Issue 1

Received 8 April 2014

Accepted 16 June 2014

Published 30 January 2015

Online at http://www.engj.org/

DOI:10.4186/ej.2015.19.1.97 


\section{Introduction}

Cancer is one of the diseases causing millions of death around the world every year. According to World Health Organization, 7.6 million people worldwide have died from cancer in 2008. In England, 410,500 people have been diagnosed with cancer in 2008 [1]. In US, 1.47 million new cancer cases have been found in 2009 [2]. From the impact of cancer, advanced algorithms have been widely studied in order to improve the treatment efficacy $[3,4]$.

In the past decades, model-based optimal control algorithms have been developed $[5,6,7]$. The aim is to compute an efficient treatment profile that satisfies a performance objective such as minimizing the tumor volume. The optimization problem is solved based on only the patient's states at the beginning of the treatment. Since these algorithms do not revise their treatment profiles according to the patient's responses during treatments, the treatment performance significantly deteriorates as the model-patient mismatch occurs.

Another technique for cancer treatment is model predictive control (MPC). MPC is an advanced control algorithm for multivariable control problem $[8,9,10]$. At each sampling instant, the patient's states are measured and a dynamic optimization problem based on the explicit model (e.g. tumor growth, pharmacokinetics and pharmacodynamics) is solved on-line. Although an optimal treatment profile is calculated, only the first computed element is implemented. Since the optimality is achieved while all of the constraints are satisfied, MPC is a promising technique for cancer chemotherapy.

A nonlinear MPC algorithm for dosing daily anticancer agents was developed by Florian et al. [11]. The full nonlinear model is incorporated in the problem formulation. The optimization problem is solved at each day to calculate an optimal dose of chemotherapeutic agents that can decrease the tumor volume along a specified reference trajectory. Since the optimization problem is formulated by using the complicated nonlinear model, the algorithm requires large on-line computational time. Moreover, the model uncertainty is not explicitly included in the problem formulation so the treatment performance deteriorates as the model-patient mismatch increases. In Chareyon and Alamir [12], the model uncertainty is handled by direct addition of a correction term. This correction term is updated based on the measured tumor volume. The uncertain parameter is not directly estimated so this method yields accurate results only in the case of the simple patient's model.

Nonlinear MPC with joint state and parameter estimation was developed by Chen et al. [1]. The moving horizon estimation method is used to estimate the uncertain parameter and the patient's states. At each sampling time, their estimated values are obtained by solving a dynamic estimation problem over a finite time horizon. These values are then used in the formulation of nonlinear MPC to compute an optimal treatment profile. The main drawback of this approach lies in the fact that both estimation problem and nonlinear control problem have to be solved at each sampling instant so the computational complexity significantly increases.

Since the model is only an approximation of the real patient, MPC should be robust to the modelpatient mismatch. In this paper, we consider an application of robust MPC to optimal dosing of breast cancer chemotherapy. At each sampling time, an optimal dose of chemotherapeutic agent is obtained by solving a convex optimization problem subject to linear matrix inequality (LMI) constraints [13]. This article is organized as follows. The mathematical model is described in Section 2. In Section 3, a robust MPC algorithm based on LMI is presented. In Section 4, the simulation results are presented. The conclusions are then drawn in section 5 .

\section{The Mathematical Model}

In this paper, we consider the problem of decreasing the tumor volume using the chemotherapeutic agent (tamoxifen). The tumor growth, the absorption of tamoxifen and the effect of tamoxifen can be described by growth kinetics, pharmacokinetics and pharmacodynamics, respectively. Figure 1 shows interactions among the five phases of the cell cycle. They are $G_{o}$ (quiescence), $G_{1}$ (growth), $S$ (DNA replication), $G_{2}$ (mitotic preparation) and $m$ (mitosis). 


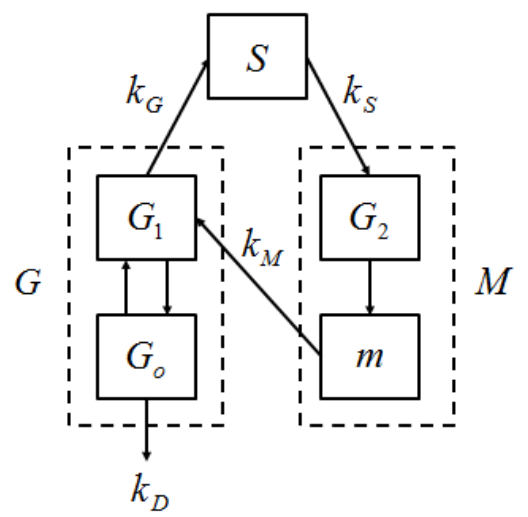

Fig. 1. The interactions among the five phases of the cell cycle.

Based on total DNA content, $G_{o}$ and $G_{1}$ phases contain the same amount of DNA so they can be lumped together into a single phase $G$. Similarly, $G_{2}$ and $m$ phases contain the same amount of DNA so they can be lumped together into a single phase $M$ [14]. The growth kinetics can be described by the following equations [11]

$$
\begin{gathered}
\frac{d X_{G}}{d t}=-k_{G} X_{G} \ln \left(\frac{\theta}{N}\right)+2 k_{M} X_{M} \ln \left(\frac{\theta}{N}\right) \\
\frac{d X_{S}}{d t}=-k_{S} X_{S}+k_{G} X_{G} \ln \left(\frac{\theta}{N}\right) \\
\frac{d X_{M}}{d t}=-k_{M} X_{M} \ln \left(\frac{\theta}{N}\right)+k_{S} X_{S}
\end{gathered}
$$

where $X_{G}$ is the volume of tumor cells in $G$ phase, $X_{S}$ is the volume of tumor cells in $S$ phase, $X_{M}$ is the volume of tumor cells in $M$ phase and $N=X_{G}+X_{S}+X_{M}$ is the total tumor volume. It is assumed that $1 \mathrm{~mm}^{3}$ is equivalent to $10^{6}$ cells. The plateau population of the tumor $\theta$ is set at $10^{4} \mathrm{~mm}^{3}$. The values of the transfer rates shown in Table 1 are obtained by minimizing the squared difference between model predictions and tumor growth [15].

Table 1. The parameters of the tumor growth model.

\begin{tabular}{lccr}
\hline Parameter & Description & Value & Unit \\
\hline$k_{G}$ & Transfer rate from $G$ phase to $S$ phase & 0.0013 & $\mathrm{~h}^{-1}$ \\
$k_{S}$ & Transfer rate from $S$ phase to $M$ phase & 0.0390 & $\mathrm{~h}^{-1}$ \\
$k_{M}$ & Transfer rate from $M$ phase to $G$ phase & 0.0169 & $\mathrm{~h}^{-1}$ \\
$\theta$ & Plateau population & $10^{4}$ & $\mathrm{~mm}^{3}$ \\
\hline
\end{tabular}

The pharmacokinetics is described by the four-compartment model as shown in Fig. 2. The two initial compartments represent the digestive tract from which tamoxifen is absorbed. After tamoxifen is absorbed into plasma, tamoxifen is converted to its metabolites such as 4-hydroxytamoxifen, N-desmethyltamoxifen, and others. Of all these metabolites, only 4-hydroxytamoxifen contributes a significant anti-tumor effect [16]. For this reason, in plasma, only tamoxifen and 4-hydroxytamoxifen are considered. The mass of tamoxifen in plasma is represented by the third compartment and the mass of 4-hydroxytamoxifen is represented by the fourth compartment.

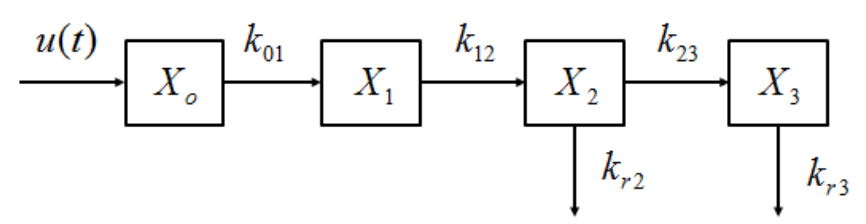

Fig. 2. The four-compartment model of the pharmacokinetics. 
The pharmacokinetics can be described by the following equations:

$$
\begin{gathered}
\frac{d X_{0}}{d t}=-k_{01} X_{0}+u(t) \\
\frac{d X_{1}}{d t}=-k_{12} X_{1}+k_{01} X_{0} \\
\frac{d X_{2}}{d t}=-k_{r 2} X_{2}-k_{23} X_{2}+k_{12} X_{1} \\
\frac{d X_{3}}{d t}=-k_{r 3} X_{3}+k_{23} X_{2} \\
Y_{1}=\frac{X_{2}}{V} \\
Y_{2}=\frac{X_{3}}{V}
\end{gathered}
$$

where $X_{0}$ is the mass of tamoxifen in the first compartment, $X_{1}$ is the mass of tamoxifen in the second

\begin{tabular}{|c|c|c|c|}
\hline Parameter & Description & Value & Unit \\
\hline$k_{01}$ & Transfer rate from $1^{\text {st }}$ compartment to $2^{\text {nd }}$ compartment & 0.048 & $\mathrm{~h}^{-1}$ \\
\hline$k_{12}$ & Transfer rate from $2^{\text {nd }}$ compartment to $3^{\text {rd }}$ compartment & 0.993 & $h^{-1}$ \\
\hline$k_{23}$ & Transfer rate from $3^{\text {rd }}$ compartment to $4^{\text {th }}$ compartment & 35.932 & $h^{-1}$ \\
\hline$k_{r 2}$ & Transfer rate from tamoxifen to other metabolites & 1.145 & $\mathrm{~h}^{-1}$ \\
\hline$k_{r 3}$ & Transfer rate from 4-hydroxytamoxifen to other metabolites & 39.525 & $\mathrm{~h}^{-1}$ \\
\hline$V$ & Compartment volume of tamoxifen and 4-hydroxytamoxifen & 8.592 & $\mathrm{~mL}$ \\
\hline
\end{tabular}
compartment, $X_{2}$ is the mass of tamoxifen in plasma, $X_{3}$ is the mass of 4-hydroxytamoxifen in plasma, $Y_{1}$ is the concentration of tamoxifen in plasma and $Y_{2}$ is the concentration of 4-hydroxytamoxifen in plasma. The values of the parameters shown in Table 2 are obtained by minimizing the squared difference between model predictions and data obtained from non-tumor-bearing athymic mice [17].

Table 2. The parameters of the pharmacokinetic model.

The pharmocodynamics is modeled by including a bilinear kill term in (1) as shown in (10)

$$
\frac{d X_{G}}{d t}=-k_{G} X_{G} \ln \left(\frac{\theta}{N}\right)+2 k_{M} X_{M} \ln \left(\frac{\theta}{N}\right)-k_{D} X_{G}\left(\frac{X_{2}}{V}+\frac{c X_{3}}{V}\right)
$$

This bilinear kill term indicates that increases in the mass of tamoxifen and 4-hydroxytamoxifen in plasma have an increasing anti-tumor effect. The parameter $c$ accounts for the different binding affinity of tamoxifen and 4-hydroxytamoxifen and it is set at 25. The value of the death rate $k_{D}=0.0062 \mathrm{~mL}^{-1} \mathrm{~g}^{-1} \mathrm{hr}^{-1}$ is obtained by minimizing the squared difference between model predictions and data obtained from tumor-bearing athymic mice [15]. The effect of chemotherapy on the patient's health is described by the circulating lymphocyte level. The equation for the number of the circulating lymphocytes can be written as [18]

$$
\frac{d C}{d t}=\alpha-\beta C-k_{C} C\left(\frac{X_{2}}{V}+b \frac{X_{3}}{V}\right)
$$

where $C$ is the number of the circulating lymphocytes, $\alpha$ is the natural generation rate of the circulating lymphocytes, $\beta$ is the natural lifespan of the circulating lymphocytes and $k_{C}$ is the killing rate. The parameter $b$ accounts for the different killing effect of tamoxifen and 4-hydroxytamoxifen. The values of these parameters are summarized in Table 3. 
Table 3. The parameters of the pharmacodynamic model.

\begin{tabular}{lccr}
\hline Parameter & Description & Value & Unit \\
\hline$k_{D}$ & Death rate & $6.20 \times 10^{-3}$ & $\mathrm{~mL}_{\mu} \mathrm{g}^{-1} \mathrm{hr}^{-1}$ \\
$c$ & Binding affinity of tamoxifen and 4-hydroxytamoxifen & 25 & - \\
$\alpha$ & Natural generation rate of the circulating lymphocytes & $1.21 \times 10^{5}$ & $\mathrm{~h}^{-1}$ \\
$\beta$ & Natural lifespan of the circulating lymphocytes & 0.012 & $\mathrm{~h}^{-1}$ \\
$k_{C}$ & Killing rate & 0.010 & ${\mathrm{~mL} \mu \mathrm{g}^{-1} \mathrm{hr}^{-1}}^{-1}$ \\
$b$ & Killing effect of tamoxifen and 4-hydroxytamoxifen & 25 & - \\
\hline
\end{tabular}

\section{A Robust MPC Algorithm Based on Linear Matrix Inequalities}

MPC is an advanced control algorithm for multivariable control problem. The basic concept of MPC is to repeatedly solve an optimal control problem subject to system dynamics, input and output constraints. MPC is a promising technique for dosing of breast cancer chemotherapy because the optimality can be achieved while all of the constraints (e.g. the upper limit for the dose of tamoxifen and the lower limit for the number of the circulating lymphocytes) can be satisfied. At each sampling instant, the patient's states are measured and an optimal dose of tamoxifen is computed on-line by solving a dynamic optimization problem based on the patient's model. Since the model is only an approximation of the real patient, MPC should be robust to the model-patient mismatch.

In this section, a robust MPC algorithm based on LMI is presented. Due to inter-patient variability and time-varying states of patient, all parameters in Tables 1,2 and 3 are considered to have a certain degree of uncertainty. Consider the following linear time-varying system with polytopic uncertainty

$$
\begin{aligned}
& x(k+1)=A(k) x(k)+B(k) u(k) \\
& y(k)=C x(k)
\end{aligned}
$$

where $x(k)$ is the vector of the patient's states, $u(k)$ is the dose of tamoxifen and $y(k)$ is the vector of the measured patient's states. The matrices $A(k)$ and $B(k)$ belong to the polytope $\Omega$

$$
[A(k), B(k)] \in \Omega, \Omega=\operatorname{Co}\left\{\left[A_{1}, B_{1}\right],\left[A_{2}, B_{2}\right], \ldots,\left[A_{L}, B_{L}\right]\right\}
$$

where $C o$ denote the convex hull and $\left[A_{j}, B_{j}\right], j=1,2, \ldots, L$ are the vertices of the convex hull. From the convexity of the polytopic description, any $A(k)$ and $B(k)$ can be written as

$$
[A(k), B(k)]=\sum_{j=1}^{L} \lambda_{j}(k)\left[A_{j}, B_{j}\right], \sum_{j=1}^{L} \lambda_{j}(k)=1,0 \leq \lambda_{j}(k) \leq 1
$$

where $\lambda(k)=\left[\lambda_{1}(k), \lambda_{2}(k), \ldots, \lambda_{L}(k)\right]$ is the uncertain parameter vector. A detailed technique to transform the nonlinear system (2)-(7), (10) and (11) to the linear time-varying system with polytopic uncertainty (12)-(14) can be found in Toth [19]. Note that the set point (e.g. the target tumor volume) is considered to be the equilibrium point in this paper.

The aim of this paper is to compute the state feedback control law $u(k+i / k)=K x(k+i / k)$ that robustly stabilizes the linear time-varying system with polytopic uncertainty (12)-(14) and achieves the following performance cost

$$
\min _{K, P} \max _{[A(k+i), B(k+i)] \in \Omega} J_{n, \infty}(k)=\sum_{i=0}^{\infty} \hat{x}(k+i / k)^{T} \hat{\Theta} \hat{x}(k+i / k)+\hat{x}(k+i / k)^{T} K^{T} R K \hat{x}(k+i / k)
$$

where $K$ is a state feedback gain, $P$ is a Lyapunov matrix, $\Theta$ and $R$ are symmetric weighting matrices, subject to

$$
\begin{gathered}
x(k / k)^{T} P x(k / k) \leq \gamma_{n}, x(k / k)=\hat{x}(k / k) \\
\hat{x}(k+i+1 / k)^{T} \hat{P} \hat{x}(k+i+1 / k)-\hat{x}(k+i / k)^{T} \hat{P} \hat{x}(k+i / k) \leq \\
\hat{-x}(k+i / k)^{T} \hat{\Theta x}(k+i / k)-\hat{x}(k+i / k)^{T} K^{T} R K \hat{x}(k+i / k) \\
x(k+i+1 / k)^{T} P x(k+i+1 / k)-x(k+i / k)^{T} P x(k+i / k) \leq 0 \\
|K x(k+i / k)| \leq \bar{u} \\
\left|(C x(k+i+1 / k))_{r}\right| \leq \bar{y}_{r}, r=1,2, \ldots, n_{y}
\end{gathered}
$$


where $\gamma_{n}$ is the upper bound on $J_{n, \infty}(k), \hat{x}(k+i+1 / k)=[\hat{A}+\hat{B} K] \hat{x}(k+i / k)$ is the vector of the nominal patient's states, $[\hat{A}, \hat{B}]=\sum_{j=1}^{L}\left[A_{j}, B_{j}\right] / L$ is the nominal patient's model, $\bar{u}$ is the limit for the dose of tamoxifen, $\bar{y}$ is the vector of the limits for patient's states and $n_{y}$ is the number of the measured patient's states. An ellipsoidal invariant set containing the measured patient's states at each sampling instant is computed by (16). By summing (17) from $i=0$ to $i=\infty$ and applying (16), it follows that $\gamma_{n}$ is the upper bound on $J_{n, \infty}(k)$. (18) is for guaranteeing that the Lyapunov function $x(k+i / k)^{T} P x(k+i / k)$ is strictly decreasing so the closed-loop system is robustly stabilized. The constraint on the dose of tamoxifen is imposed by (19). The constraints on the patient's states are imposed by (20).

By following Bumroongsri and Kheawhom [20], the constraints (16)-(20) can be written as the LMI constraints (22)-(26), respectively. At each sampling instant, the state feedback control law that minimizes the upper bound $\gamma_{n}$ on $J_{n, \infty}(k)$ and robustly stabilizes the closed-loop system is given by $u(k+i / k)=K x(k+i / k), K=Y G^{-1}$ where $Y$ and $G$ are obtained by solving the following optimization problem

$$
\begin{aligned}
& \min _{Y, G, Q_{j}} \gamma_{n} \\
& \text { s.t. }\left[\begin{array}{cc}
1 & * \\
x(k / k) & Q_{j}
\end{array}\right] \geq 0, \forall j=1,2, \ldots, L \\
& {\left[\begin{array}{cccc}
G+G^{T}-Q_{j} & * & * & * \\
\hat{A} G+\hat{B Y} & Q_{l} & * & * \\
\Theta^{\frac{1}{2}} G & 0 & \gamma_{n} I & * \\
R^{\frac{1}{2}} Y & 0 & 0 & \gamma_{n} I
\end{array}\right] \geq 0, \forall j=1,2, \ldots, L, \forall l=1,2, \ldots, L} \\
& {\left[\begin{array}{cc}
G+G^{T}-Q_{j} & * \\
A_{j} G+B_{j} Y & Q_{l}
\end{array}\right] \geq 0, \forall j=1,2, \ldots, L, \forall l=1,2, \ldots, L} \\
& {\left[\begin{array}{cc}
X & * \\
Y^{T} & G+G^{T}-Q_{j}
\end{array}\right] \geq 0, \forall j=1,2, \ldots, L, X \leq \bar{u}^{2}} \\
& {\left[\begin{array}{cc}
S & * \\
\left(A_{j} G+B_{j} Y\right)^{T} C^{T} & G+G^{T}-Q_{j}
\end{array}\right] \geq 0, \forall j=1,2, \ldots, L, S_{r} \leq \bar{y}_{r}^{2}, r=1,2, \ldots, n_{y} .}
\end{aligned}
$$

It is seen that the complicated nonlinear optimization problem is transformed to the convex optimization problem subject to LMI constraints so the computational complexity is significantly reduced.

\section{Simulation Results}

In this section, the robust MPC algorithm in Section 3 is applied to scheduling the delivery of tamoxifen to the simulated patients. The model-patient mismatch is created by subjecting all of the model parameters in Tables 1,2 and 3 to a certain deviation. The objective is to reduce the total tumor volume $N=X_{G}+X_{S}+X_{M}$ from 1,000 $\mathrm{mm}^{3}$ to $25 \mathrm{~mm}^{3}$ as rapidly as possible. The set points for $X_{G}, X_{S}$ and $X_{M}$ are $22.5 \mathrm{~mm}^{3}, 1.25 \mathrm{~mm}^{3}$ and $1.25 \mathrm{~mm}^{3}$, respectively. Note that the set point (e.g. the target tumor volume) is considered to be the equilibrium point in this paper. The symmetric weighting matrices in (15) are $\Theta$ $=\operatorname{diag}[100 ; 100 ; 100 ; 0 ; 0 ; 0 ; 0 ; 0]$ and $R=1$. The upper limit for the dose of tamoxifen is $800 \mu \mathrm{g}$. The lower limit for the number of the circulating lymphocytes is $4 \times 10^{6}$. The sampling interval is 1 day. The numerical simulations have been performed in Intel Core 2 Duo (2.53 GHz), 2 GB RAM, using SeDuMi [21] and YALMIP [22] within the Matlab R2008a environment.

Figure 3 shows the total tumor volume as a function of time. Starting from the initial value of 1,000 $\mathrm{mm}^{3}$, the total tumor volume can be reduced to the specified target of $25 \mathrm{~mm}^{3}$ in the presence of up to $30 \%$ model-patient mismatch. However, the treatment result is more favorable as the level of the model-patient 
mismatch decreases. In the presence of the model-patient mismatch, the tumor volume can be reduced because model uncertainty is explicitly included in the problem formulation.

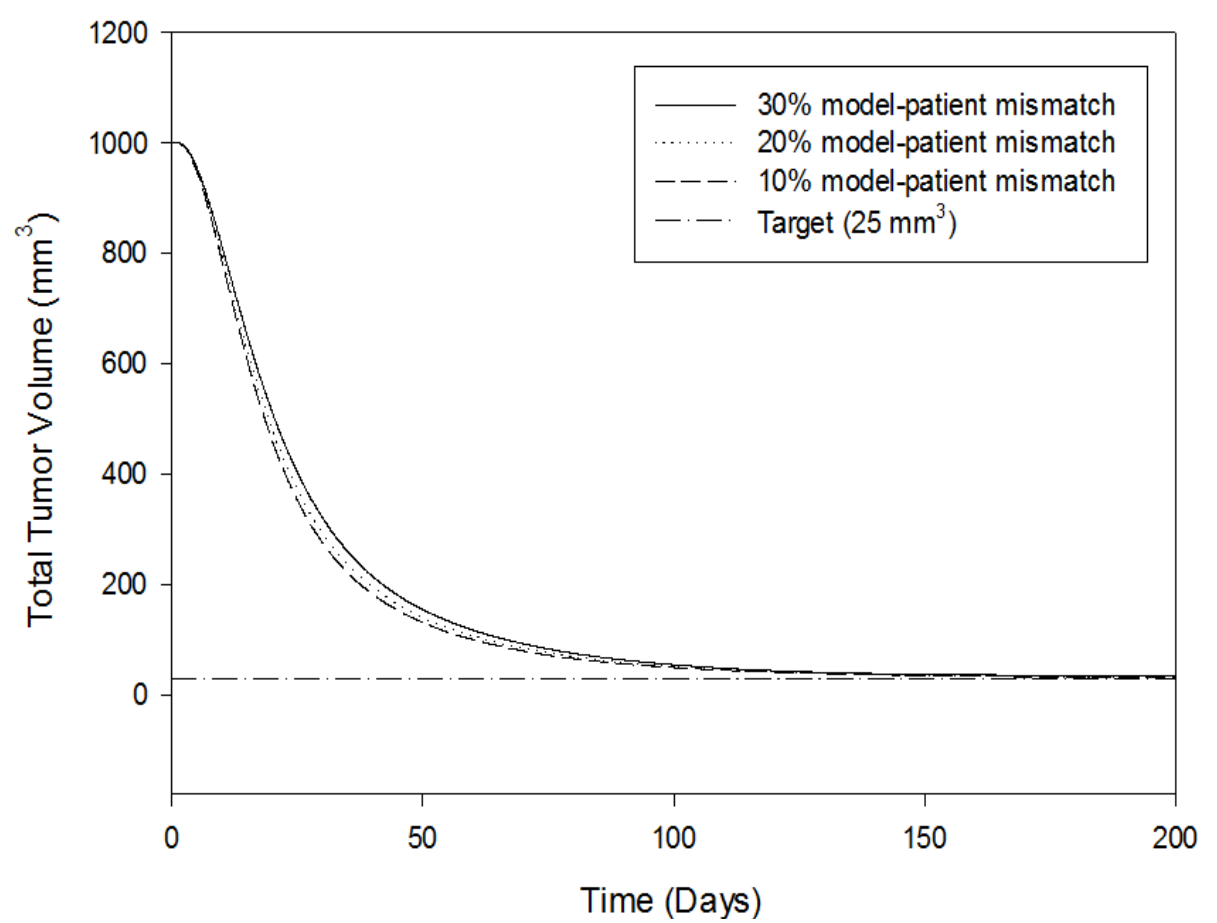

Fig. 3. The total tumor volume as a function of time computed by the robust MPC algorithm.

Figure 4 shows the dose of tamoxifen as a function of time. The dose of tamoxifen decreases as the level of model-patient mismatch increases. However, the dose of tamoxifen converges to its nominal value for all levels of the model-patient mismatch.

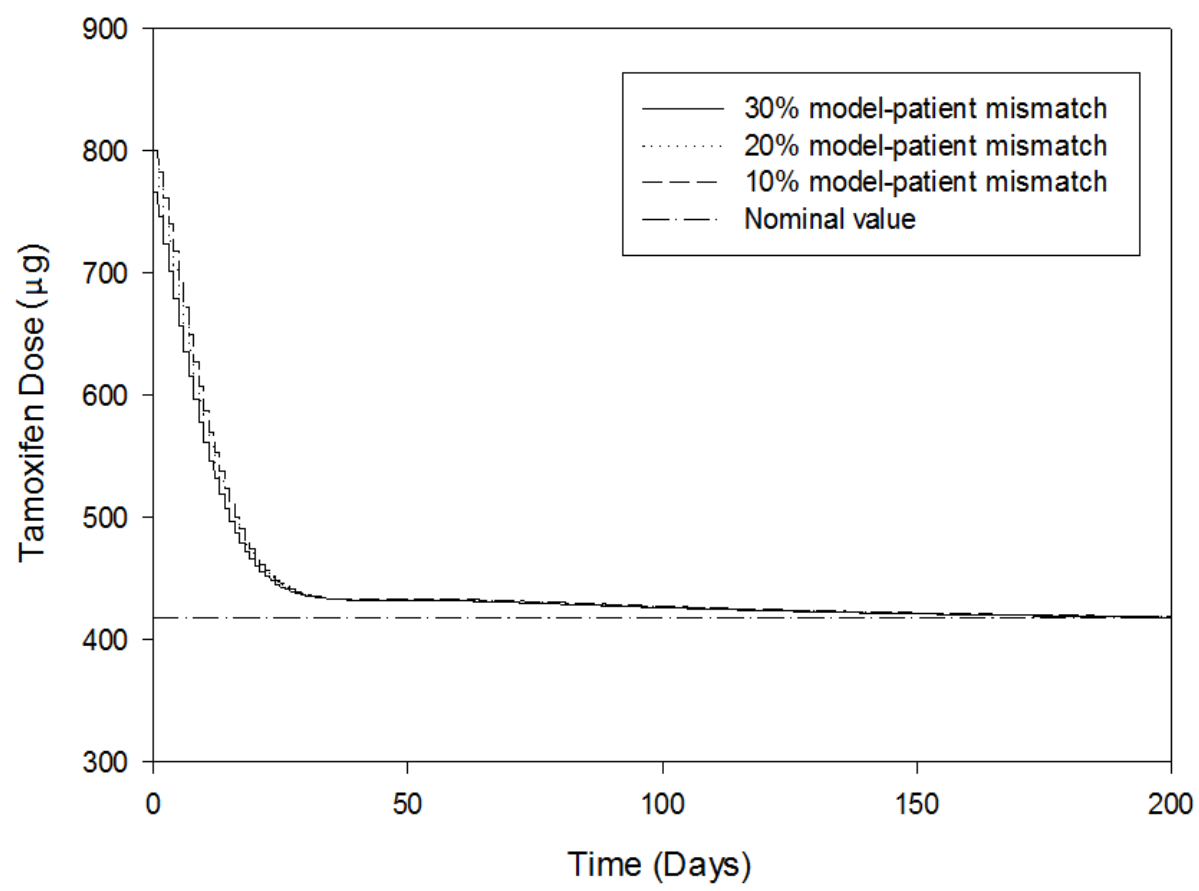

Fig. 4. The dose of tamoxifen as a function of time computed by the robust MPC algorithm.

The robust MPC algorithm will be compared with the nonlinear MPC algorithm [11] where the nonlinear system (2)-(7), (10) and (11) is directly used in the problem formulation. The resulting nonlinear optimization problem is solved by using the fmincon function in Matlab R2008a. The weighting matrices of 
nonlinear MPC are the same as those of robust MPC. The prediction horizon and the control horizon are 5 days and 2 days, respectively. Figure 5 shows the total tumor volume as a function of time. It is seen that the nonlinear MPC algorithm cannot reduce the total tumor volume to the specified target of $25 \mathrm{~mm}^{3}$ in the presence of $30 \%$ model-patient mismatch. For the nonlinear MPC algorithm, the model uncertainty is not explicitly included in the problem formulation so the tumor volume cannot be reduced to a specified target.

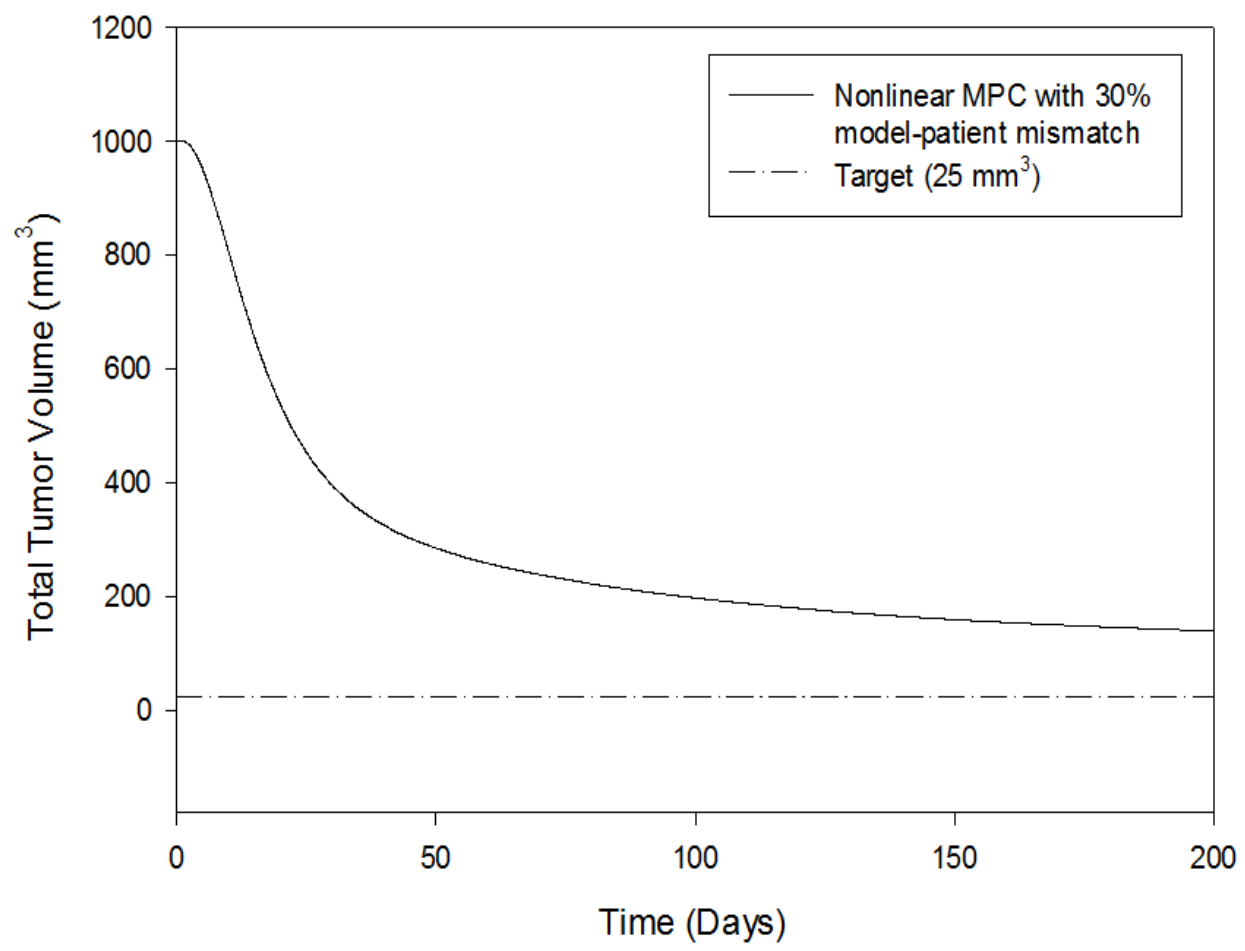

Fig. 5. The total tumor volume as a function of time computed by the nonlinear MPC algorithm.

Figure 6 shows the dose of tamoxifen as a function of time. It is seen that for the nonlinear MPC algorithm, the dose of tamoxifen does not converge to its nominal value.

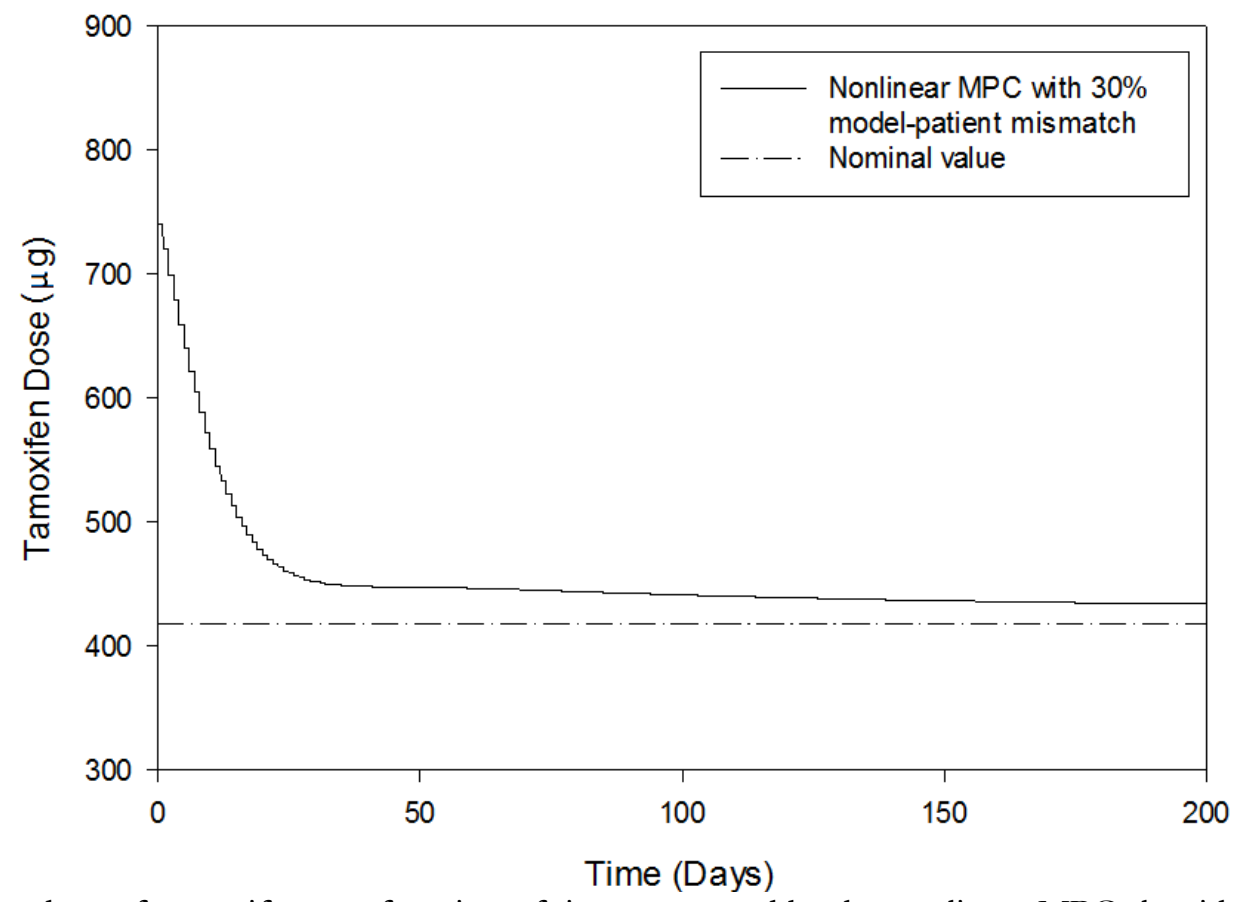

Fig. 6. The dose of tamoxifen as a function of time computed by the nonlinear MPC algorithm. 
The on-line computational burden is shown in Table 4. It can be observed that the robust MPC algorithm requires less on-line computational time that the nonlinear MPC algorithm. This is due to the fact that the nonlinear optimization problem is transformed to the convex optimization problem subject to LMI constraints so the computational complexity is significantly reduced. The main drawback of the nonlinear MPC algorithm is that it would be numerically intractable for large prediction and control horizons.

Table 4. The on-line computational burden.

\begin{tabular}{lr}
\hline Algorithm & CPU time (s) per step \\
\hline The robust MPC algorithm & 120 \\
The nonlinear MPC algorithm & 1,800 \\
\hline
\end{tabular}

\section{Conclusions}

In this research, we have presented an application of robust MPC algorithm to optimal dosing of breast cancer chemotherapy. The model uncertainty is explicitly included in the problem formulation. At each sampling time, an optimal dose of chemotherapeutic agent is obtained by solving a convex optimization problem subject to linear matrix inequality constraints. A case study of simulated patients is presented. In the presence of the model-patient mismatch, the robust MPC algorithm can achieve better treatment results than the nonlinear MPC algorithm while the computational complexity is significantly reduced.

\section{Acknowledgements}

This research project is supported by Mahidol University.

\section{References}

[1] T. Chen, N. F. Kirkby, and R. Jena, "Optimal dosing of cancer chemotherapy using model predictive control and moving horizon state/parameter estimation," Comput. Meth. Prog. Bio., vol. 108, pp. 973 983, 2012.

[2] K. L. Kiran and S. Lakshminarayanan, "Global sensitivity analysis and model-based reactive scheduling of targeted cancer immunotherapy," Biosystems, vol. 101, pp. 117-126, 2010.

[3] A. Cappuccio, F. Castiglione, and B. Piccoli, "Determination of the optimal therapeutic protocols in cancer immunotherapy," Math. Biosci., vol. 209, pp. 1-13, 2007.

[4] P. Dua, V. Dua, and E. Pistikopoulos, "Optimal Delivery of chemotherapeutic agents in cancer," Comput. Chem. Eng., vol. 32, pp. 99-107, 2008.

[5] S. M. Tse, Y. Liang, K. S. Leung, K. H. Lee, and T. K. Mok, "A memetic algorithm for multiple-drug cancer chemotherapy schedule optimization," IEEE T. Syst. Man Cyb., vol. 37, pp. 84-91, 2007.

[6] A. Ghaffari and N. Naserifar, "Optimal therapeutic protocols in cancer immunotherapy," Comput. Biol. Med., vol. 40, pp. 261-270, 2010.

[7] M. Engelhart, D. Lebiedz, and S. Sager, "Optimal control for selected cancer chemotherapy ODE models: A view on the potential of optimal schedules and choice of objective function," Math. Biosci., vol. 229, pp. 123-134, 2011.

[8] S. J. Qin and T. A. Badgwell, "A survey of industrial model predictive control technology," Control Eng. Pract., vol. 11, pp. 733-764, 2003.

[9] J. B. Rawlings and D. Q. Mayne, "Getting started with model predictive control," in Model Predictive Control: Theory and Design, $1^{\text {st }}$ ed. Wisconsin: Nob Hill Publishing, 2009, ch. 1, pp. 1-60.

[10] J. H. Lee, "Model predictive control: Review of the three decades of development," Int. J. Control Autom., vol. 9, pp. 415-424, 2011.

[11] J. A. Florian, J. L. Eiseman, and R. S. Parker, "Nonlinear model predictive control for dosing daily anticancer agents using a novel saturating-rate cell-cycle model," Comput. Biol. Med., vol. 38, pp. 339_ $347,2008$.

[12] S. Chareyron and M. Alamir, "Mixed immunotherapy and chemotherapy of tumors: Feedback design and model updating schemes," J. Theor. Biol., vol. 258, pp. 444-454, 2009. 
[13] S. Boyd and L. Vandenberghe, "Convex optimization problems," in Convex Optimization, 1st ed. Cambridge: Cambridge University Press, 2004, ch. 4, pp. 127-189.

[14] G. S. Lee, K. S. Ryu, J. G. Rha, S. P. Kim, S. E. Namkoong, and K. T. Han, "Multiparametric flow cytometric analysis in a breast cancer cell line (MCF-7)," J. Obstet. Gynaecol. Res., vol. 28, pp. 141-148, 2002.

[15] B. A. Conley, T. S. Ramsland, D. L. Sentz, S. Wu, D. M. Rosen, M. Wollman, and J. L. Eiseman, "Antitumor activity, distribution, and metabolism of 13-cis-retinoic acid as a single agent or in combination with tamoxifen in established human MCF-7 xenografts in mice," Cancer Chemother. Pharmacol., vol. 43, pp. 183-197, 1999.

[16] B. A. Chabner and D. L. Longo, "Hormonal therapy of breast cancer," in Cancer Chemotherapy and Biotherapy: Principles and Practice, $5^{\text {th }}$ ed. Philadelphia: Lippincott Williams and Wilkins, 2010, ch. 35, pp. 649-673.

[17] S. P. Robinson, F. S. M. Langan, D. A. Johnson, and V. C. Jordan, "Metabolites, pharmacodynamics, and pharmacokinetics of tamoxifen in rats and mice compared to the breast cancer patient," Drug Metab. Dispos., vol. 1, pp. 36-43, 1991.

[18] L. G. Pillis, W. Gu, and A. Radunskaya, "Mixed immunotherapy and chemotherapy of tumors: modeling, applications and biological interpretation," J. Theor. Biol., vol. 238, pp. 841-862, 2006.

[19] R. Toth, "LPV modeling of physical systems," in Modeling and Identification of Linear Parameter-Varying Systems, $1^{\text {st }}$ ed. London: Springer, 2010, ch. 7, pp. 171-195.

[20] P. Bumroongsri and S. Kheawhom, "Robust constrained MPC based on nominal performance cost with applications in chemical processes," Procedia Engineering, vol. 42, pp. 1561-1571, 2012.

[21] J. F. Sturm, "Using SeDuMi 1.02, a Matlab toolbox for optimization over symmetric cones," Optim. Method Softw., vol. 11, pp. 625-653, 1999.

[22] J. Löfberg, “Automatic robust convex programming," Optim. Method Softw., vol. 27, pp. 115-129, 2012. 